\title{
CARACTÉRISATION ÉPIDÉMIOLOGIQUE DES CAS DE SYPHILIS CONGÉNITALE DANS LE NORD DU BRÉSIL, DE 2014 À 2019
}

\section{ARTICLE ORIGINAL}

ALMEIDA, Carolina Gomes ${ }^{1}$, ÁVILA, Gabriel Pereira², TEIXEIRA, Isabelly Montenegro $^{3}$, RODRIGUES, Raíza Júlia Viana ${ }^{4}$, DIAS, Claudio Alberto Gellis de Mattos $^{5}$, OLIVEIRA, Euzébio de ${ }^{6}$, DENDASCK, Carla Viana ${ }^{7}$, ARAÚJJO, Maria Helena Mendonça de ${ }^{8}$, FECURY, Amanda Alves ${ }^{9}$

\section{ALMEIDA, Carolina Gomes. Et al. Caractérisation épidémiologique des cas de} syphilis congénitale dans le nord du Brésil, de 2014 à 2019. Revista Científica Multidisciplinar Núcleo do Conhecimento. An 05, Ed. 12, vol. 14, p. 20 à 31. décembre 2020.

ISSN:

2448-0959, Lien

d'accès:

https://www.nucleodoconhecimento.com.br/sante/dans-le-nord, DOI: 10.32749/nucleodoconhecimento.com.br/sante/dans-le-nord

\section{RÉSUMÉ}

La syphilis est une maladie causée par la bactérie Treponema pallidum, acquise, en grande partie, par transmission sexuelle. La syphilis congénitale est une maladie

\footnotetext{
${ }^{1}$ Universitaire du cours de médecine de I'Université fédérale du Pará (UNIFAP).

${ }^{2}$ Universitaire du cours de médecine de l'Université fédérale du Pará (UNIFAP).

${ }^{3}$ Universitaire du cours de médecine de l'Université fédérale du Pará (UNIFAP).

${ }^{4}$ Universitaire du cours de médecine de l'Université fédérale du Pará (UNIFAP).

${ }^{5}$ Doctorat en recherche théorique et comportementale (UFPA). Professeur et chercheur à l'Institut fédéral d'Amapá (IFAP), campus de Macapá, AP. Professeur et chercheur au Programme d'études supérieures en éducation professionnelle et technologique (ProfEPT), IFAP, campus de Santana.

${ }^{6}$ Doctorat en maladies tropicales (UFPA). Professeur et chercheur à l'Université fédérale du Pará (UFPA), Campus Castanhal, PA.

${ }^{7}$ Théologien, Ph.D. en psychanalyse clinique. II travaille depuis 15 ans avec la méthodologie scientifique (méthode de recherche) dans le cadre de l'orientation de production scientifique des étudiants à la maîtrise et au doctorat. Spécialiste de l'étude de marché et de la recherche axée sur la santé. Doctorant en Communication et Sémiotique (PUC SP).

${ }^{8}$ Master en enseignement et sciences de la santé. Professeur et chercheur à l'Université fédérale d'Amapá (UNIFAP), campus de Macapá, AP.

${ }^{9}$ Doctorat en maladies tropicales (UFPA). Professeur et chercheur à l'Université fédérale d'Amapá (UNIFAP), campus de Macapá. Professeur et chercheur au Programme d'études supérieures en sciences de la santé (UFPA). Pro-recteur de la recherche et des études supérieures à l'Université fédérale d'Amapá (UNIFAP).

$\mathrm{RC}: 96425$

Lien d'accès: https://www.nucleodoconhecimento.com.br/sante/dans-le-nord
} 
contagieuse de transmission verticale (de la mère au fœtus). Cette étude visait à présenter le nombre de cas signalés de syphilis congénitale dans le nord du Brésil, entre 2014 et 2019, et à caractériser le profil épidémiologique des cas. Des recherches descriptives, transversales et rétrospectives ont été effectuées à l'aide de la base de données DATASUS. II y a eu une augmentation du nombre de cas au cours de la période évaluée, en particulier dans l'État d'Amazonas, qui a présenté le plus grand nombre de notifications. En ce qui concerne les soins prénataux, en moyenne quatre fois plus de femmes que celles qui n'en ont pas fait. Les femmes dont la classe maternelle était de la 5 e à la 8 e école élémentaire incomplète avaient un plus grand nombre de cas de la maladie. Bien que la région du Nord ait eu une performance prénatale élevée, la plupart des cas de syphilis congénitale n'ont été diagnostiqués qu'après l'accouchement, ce qui indique des interprétations erronées concernant les tests et une erreur conséquente dans le diagnostic et le traitement. Étant donné que la forme précoce (émergence jusqu'à la 2ème année de vie) est la majorité du nombre de cas, il y a une évolution favorable de la maladie.La scolarité et le revenu semblent être des facteurs qui influencent le diagnostic tardif de la maladie. II est nécessaire d'augmenter le nombre de partenaires traités, ce qui réduit la transmission de la syphilis et, par conséquent, de la syphilis congénitale.

Mots-clés : Syphilis congénitale, Région du Nord, Prénatal.

\section{INTRODUCTION}

La syphilis est une infection bactérienne systémique, chronique, curable et exclusive de l'être humain. Lorsqu'elle n'est pas traitée, elle évolue vers des stades de gravité variable et peut affecter les organes et les systèmes du corps (BRASIL, 2018). L'agent causal de la syphilis est la bactérie Treponema pallidum, un spirochète, acquis dans la plupart des cas pendant les rapports sexuels. La maladie est classée, selon ses différentes voies de transmission, dans la syphilis acquise et la syphilis congénitale (CAVALCANTE et al., 2012, ROCHA et al., 2020).

La syphilis congénitale est le résultat d'une propagation hématogène de T. pallidum, de la femme enceinte infectée qui n'est pas traitée ou insuffisamment traitée pour son $\mathrm{RC}: 96425$

Lien d'accès: https://www.nucleodoconhecimento.com.br/sante/dans-le-nord 
conceptus, par voie transplacentérale (CRT, 2016). La transmission peut survenir tout au long de la grossesse, et le risque est plus élevé chez les femmes enceintes atteintes de syphilis primaire ou secondaire (GUINSBURG; SANTOS, 2010, BARROS et al., 2020).

C'est une maladie responsable d'environ $40 \%$ des taux de mortalité périnatale, de 25 $\%$ des mortinaissances, de $14 \%$ des décès néonatals (CARDOSO et al., 2018). En outre, il est capable d'entraîner de graves conséquences pour le conceptus en tant que complications systémiques, que ce soit dans la syphilis congénitale précoce (atteinte osseuse, neurologique, hématologique et hépatosplénique), ou tardive (stigmates résultant du remodelage et des déformations osseuses) (MOTTA et al., 2018).

Le contrôle efficace de la syphilis a pour prémisse fondamentale le dépistage sérologique et le traitement approprié des femmes enceintes et des partenaires sexuels, car la qualité des soins prénatals et de l'accouchement est un déterminant important pour réduire la transmission verticale. La pénicilline est le médicament de choix dans le traitement de la syphilis et le seul indiqué pour les femmes enceintes: il a une efficacité de $98 \%$ dans la prévention de la syphilis congénitale, agissant à tous les stades de la maladie (CAVALCANTE; PEREIRA CASTRO, 2017).

\section{OBJECTIF}

Présenter le nombre de cas signalés de syphilis congénitale dans le nord du Brésil, entre 2014 et 2019 , et caractériser le profil épidémiologique des cas.

\section{MÉTHODE}

Une étude quantitative descriptive, transversale et rétrospective a été menée à l'échelle des données du Système d'information sur les maladies à déclaration obligatoire (SINAN/SUS) du Département d'informatique du Système unifié de santé (DATASUS). Les données nationales ont été collectées selon les étapes suivantes: Le lien http://indicadoressifilis.aids.gov.br a été consulté et dans "Couverture des 
données ", "Données régionales et nationales » ont été sélectionnées et dans "Sous-catégorie " «Brésil ». Le bouton "Télécharger les données » a été cliqué et les données concernant la syphilis congénitale au Brésil ont été supprimées. Par la suite, dans "Couverture des données », "Données d'État » a été sélectionné et dans "Sous-catégorie », nous avons sélectionné le nom de chaque État de la région nord du Brésil, les tableaux de chaque État ont été téléchargés et les informations sur la syphilis congénitale ont été supprimées. Les données ont été compilées dans l'application Excel, un composant de la suite Microsoft Corporation Office.

\section{RÉSULTATS}

Le pourcentage de cas de syphilis congénitale selon le diagnostic final par année de diagnostic au Brésil entre 2014 et 2019 est indiqué dans le tableau 1. Le pourcentage de cas a augmenté, passant de 12,28 \% en 2014 à 18,21 \% en 2019.

Le tableau 1 - Montre le pourcentage de cas de syphilis congénitale selon le diagnostic final par année de diagnostic, dans le nord du Brésil, entre 2014 et 2019.

\begin{tabular}{rrc}
\hline ANO & No CASOS & $\%$ \\
\hline $\mathbf{2 0 1 4}$ & 16353 & $12.28 \%$ \\
$\mathbf{2 0 1 5}$ & 19712 & $14.80 \%$ \\
$\mathbf{2 0 1 6}$ & 21330 & $16.01 \%$ \\
$\mathbf{2 0 1 7}$ & 25037 & $18.79 \%$ \\
$\mathbf{2 0 1 8}$ & 26531 & $19.92 \%$ \\
$\mathbf{2 0 1 9}$ & 24253 & $18.21 \%$ \\
\hline
\end{tabular}

Dans le nord du Brésil, le pourcentage de cas de syphilis congénitale selon le diagnostic final par année de diagnostic entre 2014 et 2019 était de 11 071. Au cours de la période, le nombre de cas a augmenté, passant de $11,13 \%$ en 2014 à $20,19 \%$ en 2019 (figure 1). 
Figure 1 - Montre le pourcentage de cas de syphilis congénitale selon le diagnostic final par année de diagnostic, dans le nord du Brésil, entre 2014 et 2019.

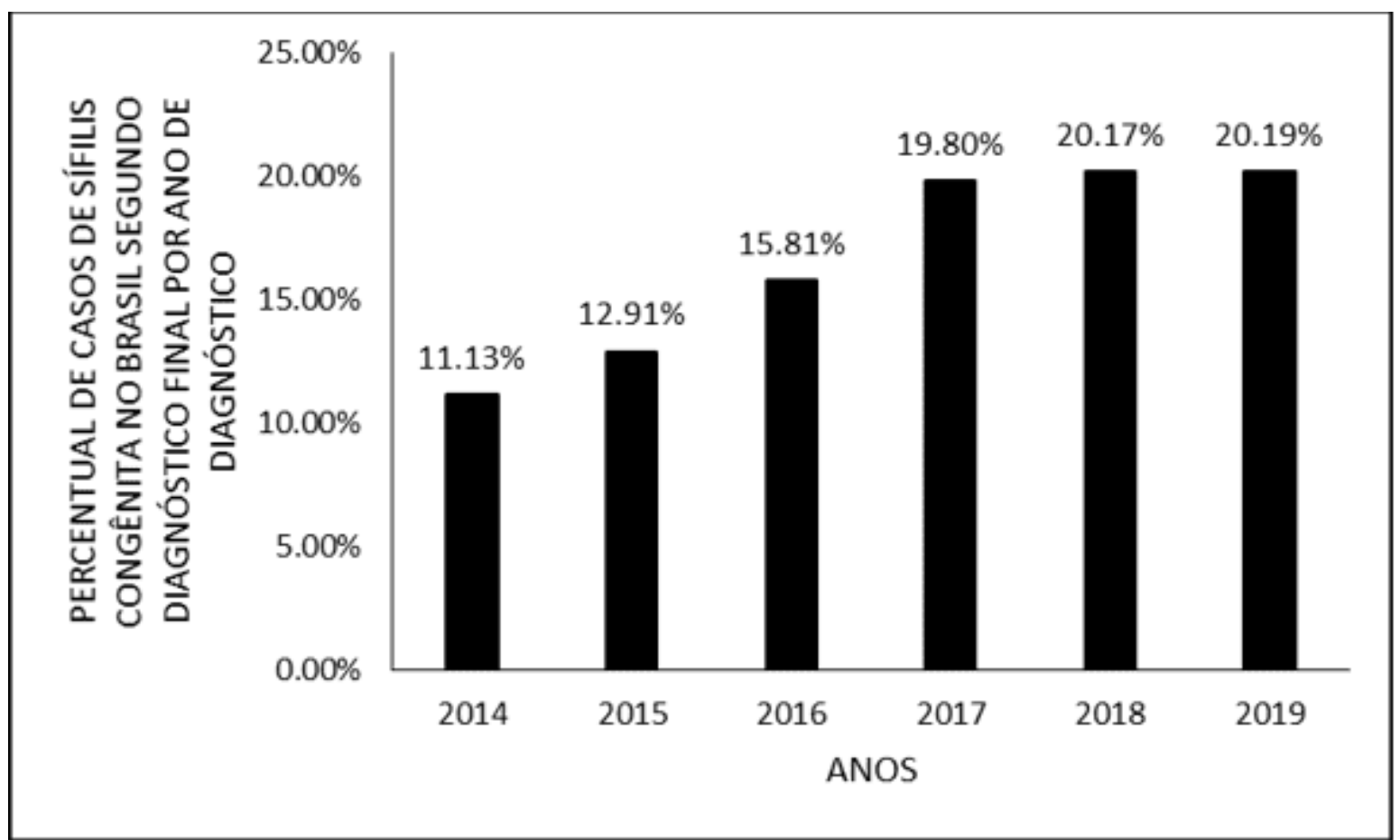

Parmi les États de la région Nord, le Pará s'est distingué avec le plus grand nombre de cas $(42,08 \%)$ et celui avec le nombre le plus faible, l'État de Roraima $(1,83 \%)$. (Figure 2). 
La figure 2 - Montre le pourcentage de cas de syphilis congénitale selon le diagnostic final par année de diagnostic, par État dans la région nord du Brésil, entre 2014 et 2019.

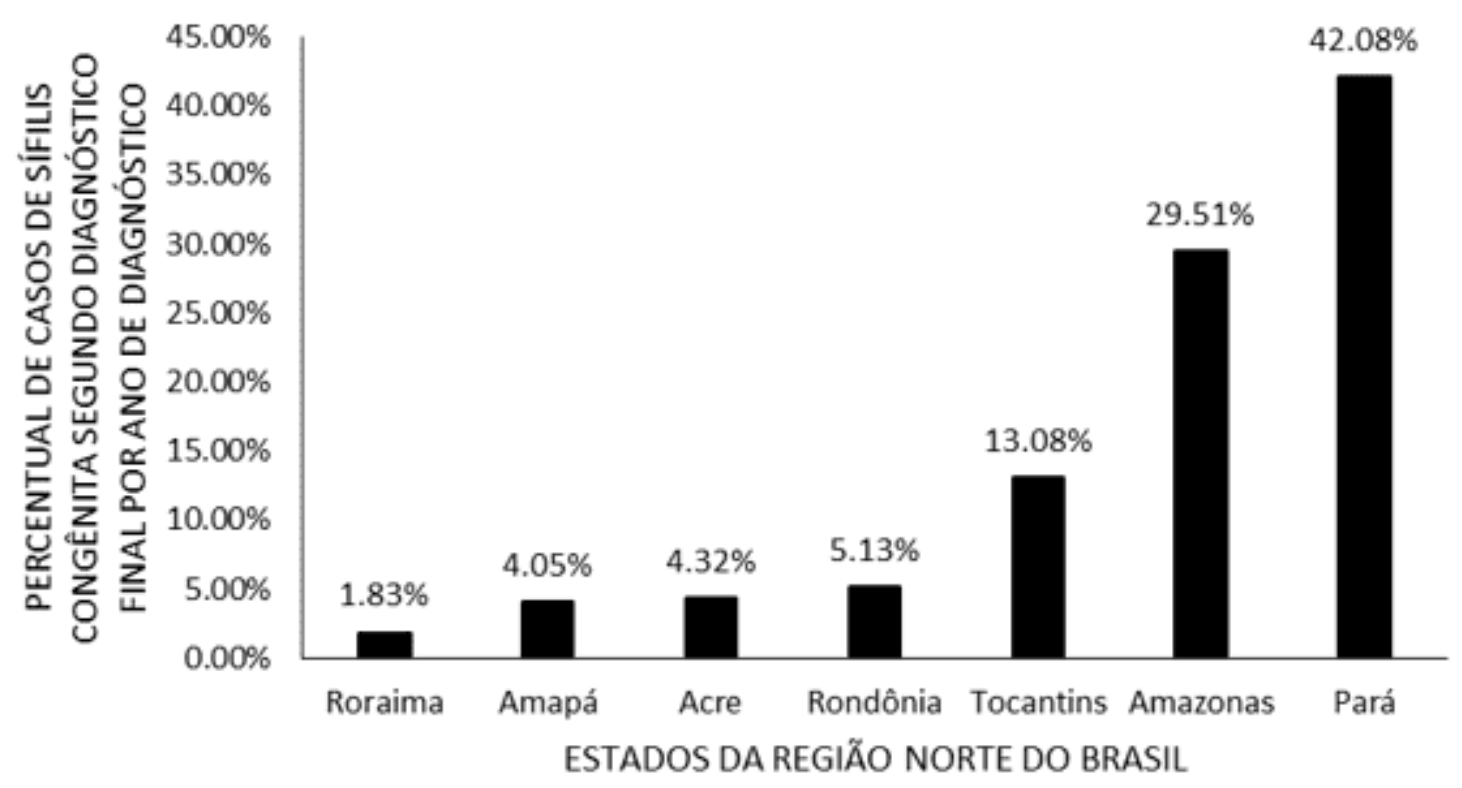

La figure 3 montre la répartition en pourcentage des cas de syphilis congénitale dans la région du Nord par deuxième race/couleur (origine ethnique) entre 2014 et 2019. Les données indiquent un nombre plus élevé de cas dans le groupe ethnique brun avec 9638 (87,06\%), suivi par les blancs avec 608 (5,49\%). Les groupes ethniques ayant le nombre le plus faible étaient les Autochtones avec $85(0,77 \%)$ et les Noirs avec $32(0,29 \%)$, respectivement. 
Figure 3 - Montre la répartition en pourcentage des cas de syphilis congénitale dans la région du Nord par deuxième race/couleur (origine ethnique) entre 2014 et 2019.

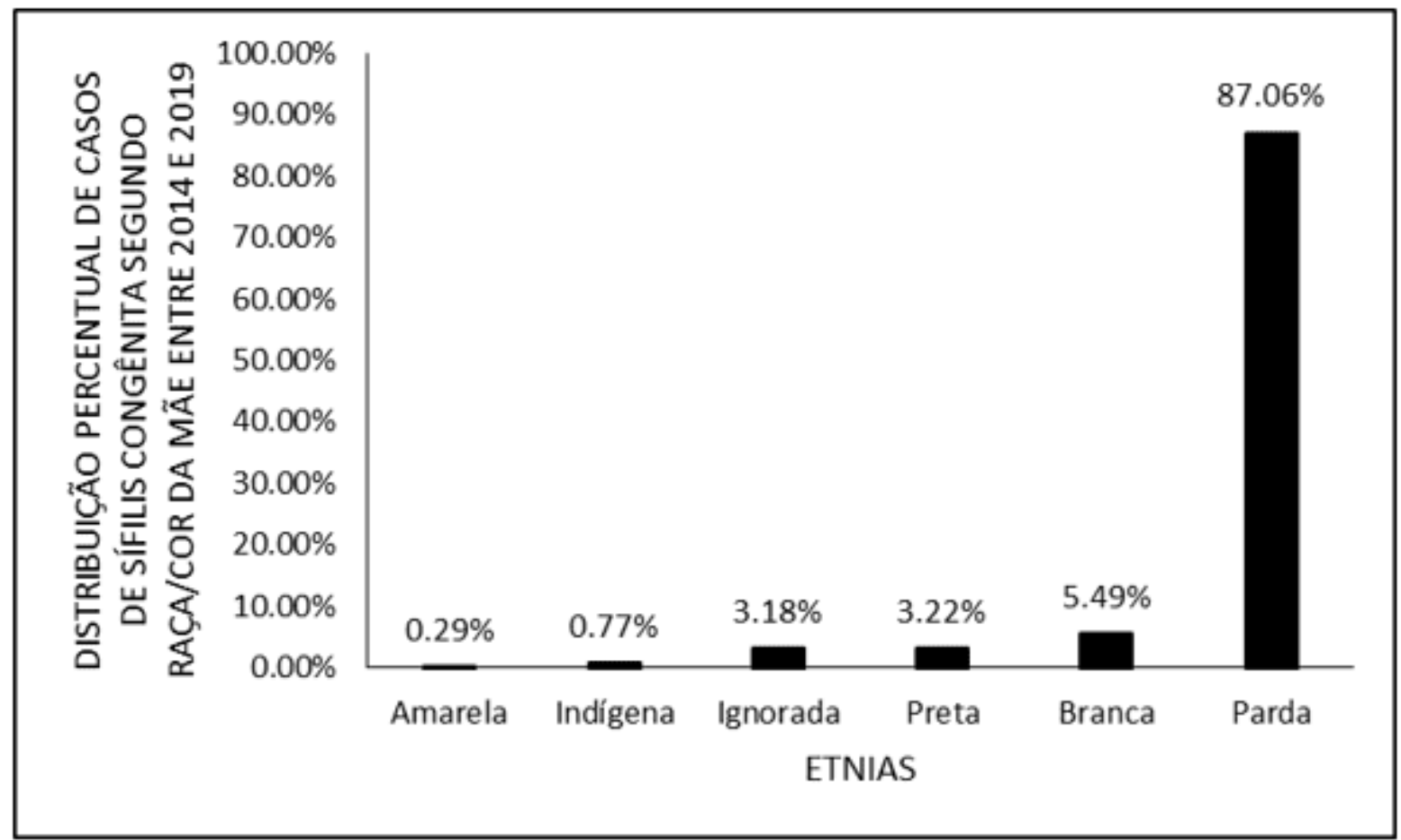

La figure 4 montre le nombre de cas confirmés de syphilis congénitale dans la région du Nord par examen prénatal entre 2014 et 2019. Les données montrent qu'un plus grand nombre de femmes qui ont subi des soins prénatals 8712 (78,69\%) que celles qui n'ont pas subi $2048(18,50 \%)$ et celles considérées comme ignorées totalisaient $311(2,81 \%)$. 
Figure 4 - montre la répartition en pourcentage des cas de syphilis congénitale selon les informations sur les soins prénataux de la mère par année de diagnostic entre 2014 et 2019.

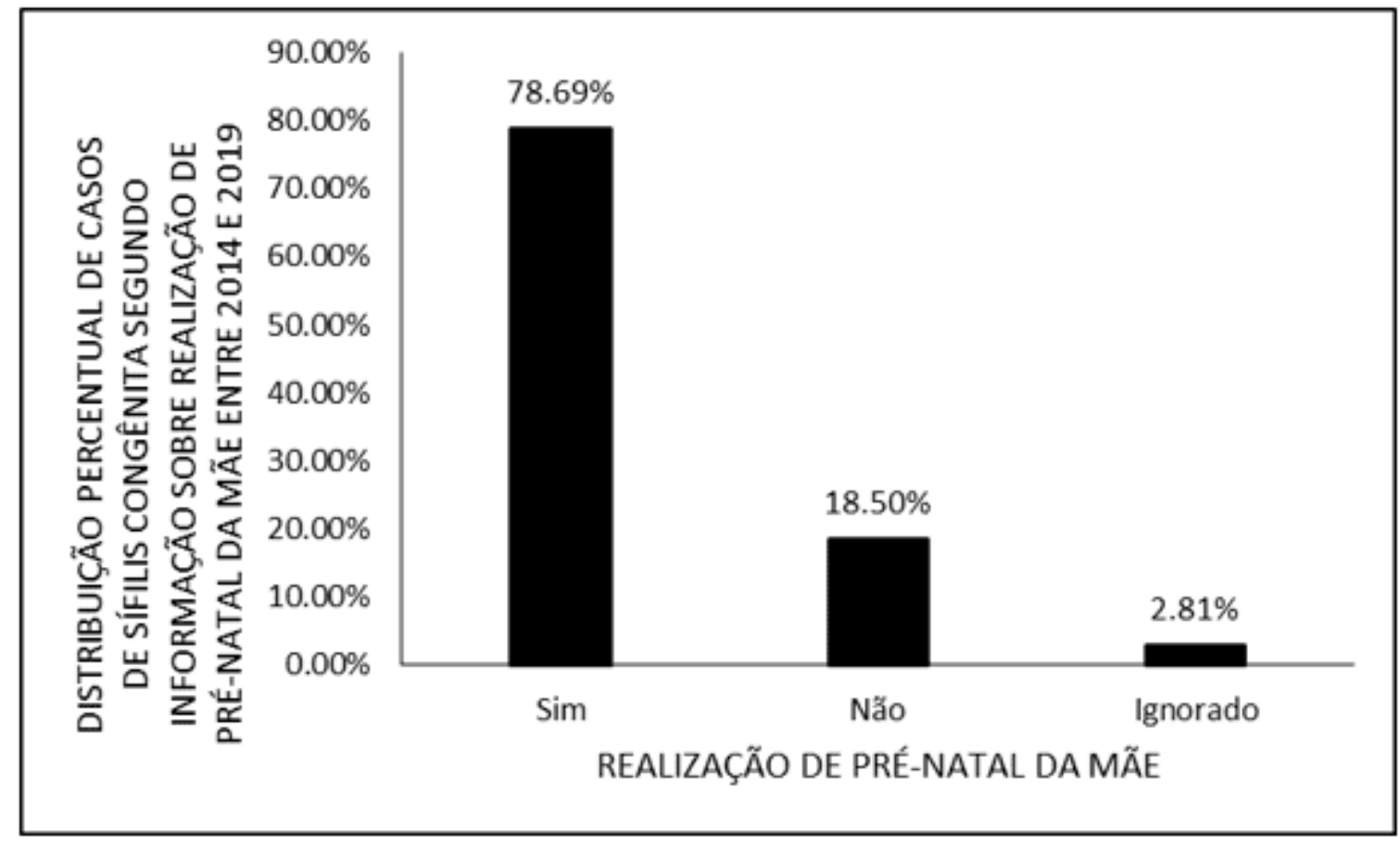

La figure 5 montre la répartition en pourcentage des cas de syphilis congénitale dans la région du Nord, selon la scolarité de la mère par année de diagnostic entre 2014 et 2019. Les données montrent un nombre important de mères analphabètes de 62 femmes $(0,90 \%)$, la majorité des cas avec la classe maternelle de la $5 e$ à la $8 \mathrm{e}$ école élémentaire incomplète avec $2.362(33,80 \%)$, avec une collection de femmes ayant terminé l'école primaire de $936(13,40 \%)$ et une partie ayant terminé leurs études secondaires $1.320(18,90 \%)$, le plus faible nombre de mères ayant terminé leurs études supérieures était de $62(0,90 \%)$ et le nombre de mères ignorées était de 2.243 $(32,10 \%)$. 
Figure 5 - Montre la répartition en pourcentage des cas de syphilis congénitale dans la région du Nord, selon la scolarité de la mère par année de diagnostic entre 2014 et 2019.

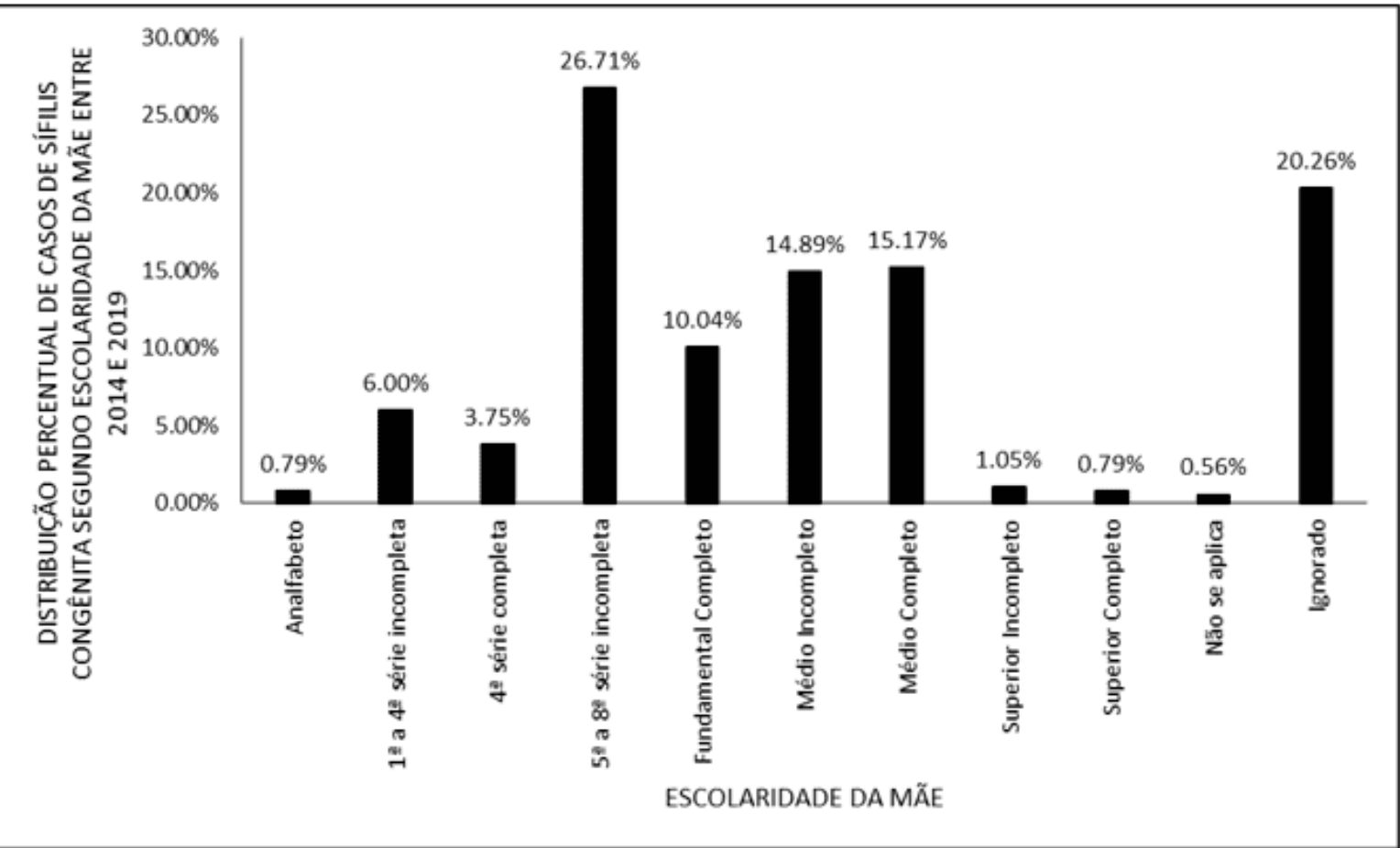

La figure 6 montre le nombre de cas confirmés de syphilis congénitale dans la région du Nord au moment du diagnostic maternel de syphilis, entre 2014 et 2019. II y a un plus grand nombre d'infections lors de l'examen prénatal avec un nombre total de cas de 4862 (43,92\%) et aussi au moment de l'accouchement avec 4258 (39,46\%), avec une minorité de cas diagnostiqués dans la période post-partum, avec un total de 1388 $(12,54 \%)$ et ceux ignorés totalisé 447 (4,04\%). 
Figure 6 - Montre la répartition en pourcentage des cas confirmés de syphilis congénitale selon le moment du diagnostic dans la région nord, entre les années 2014 et 2019.

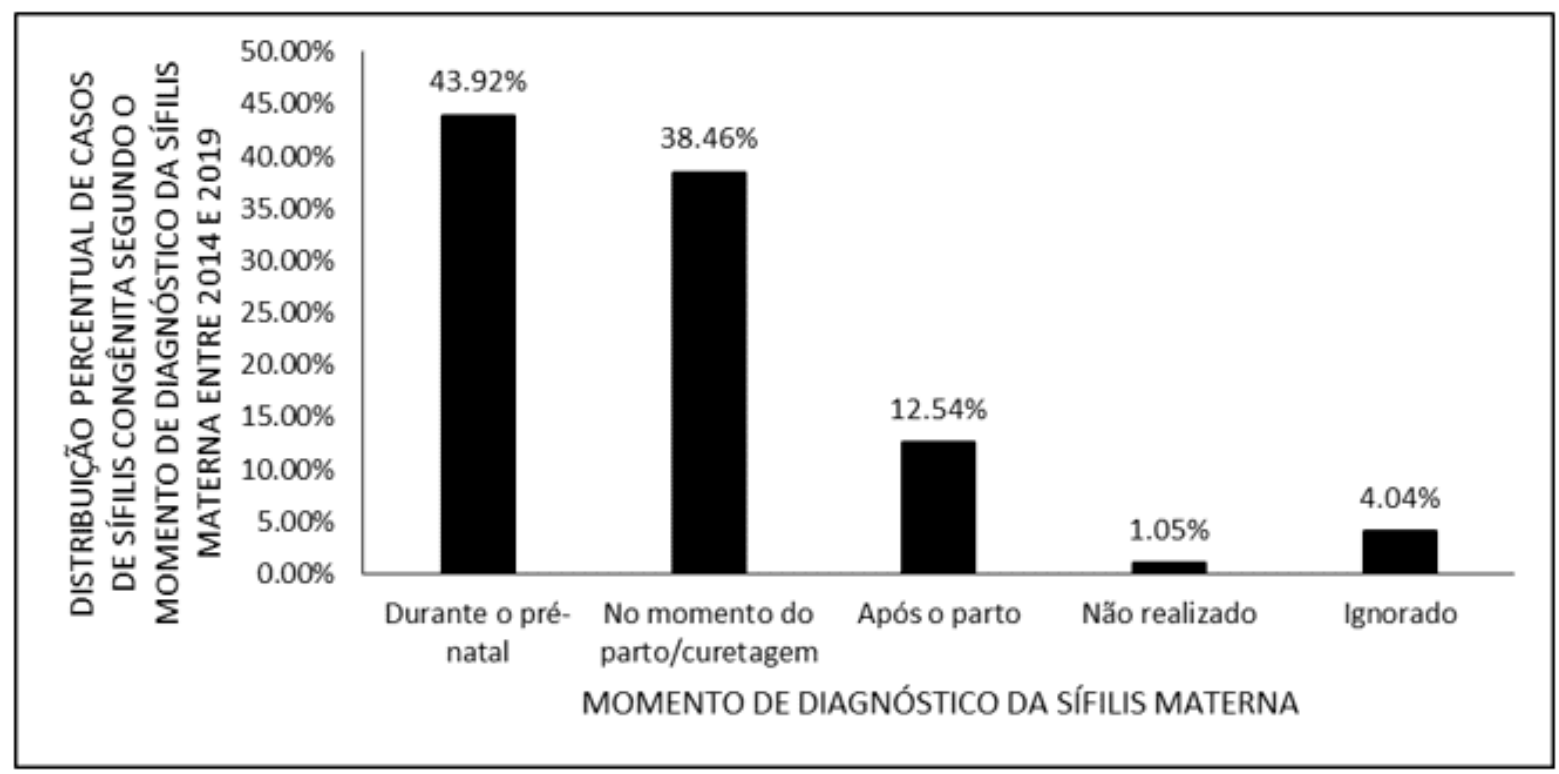

En ce qui concerne la classification finale variable de la syphilis congénitale, les données trouvées étaient 10475 (94,62\%) classées comme syphilis congénitale récente; 24 classés comme syphilis congénitale tardive (0,22\%); $256(2,31 \%)$ avortements dus à la syphilis; et 316 (2,85\%) mort-nés par syphilis (tableau 2). 
Tableau 2 - Montre la répartition en pourcentage des cas de syphilis congénitale selon le diagnostic final par année de diagnostic, dans la région nord du Brésil, entre 2014 et 2019.

\begin{tabular}{c|c|c}
\cline { 2 - 3 } & $\begin{array}{c}\text { No CASOS } \\
\text { (2014 a 2019) }\end{array}$ & $\%$ \\
\hline $\begin{array}{c}\text { Sífilis congênita } \\
\text { recente }\end{array}$ & 10475 & $94.62 \%$ \\
Sífilis congênita tardia & 24 & $0.22 \%$ \\
Aborto por sífilis & 256 & $2.31 \%$ \\
Natimorto por sífilis & 316 & $2.85 \%$ \\
\hline
\end{tabular}

\section{DISCUSSION}

Il y a eu une augmentation du nombre de cas de 2014 à 2019 (figure 1), en particulier dans l'État d'Amazonas, qui a augmenté de 416\% (figure 2) les enregistrements de notification de la maladie au cours de la période observée, étant même l'État de la région du Nord avec le PIB par habitant le plus élevé (IBGE, 2016). Cette analyse de la relation entre l'incidence de la maladie et la capacité économique du site est faite par le fait que la syphilis congénitale est une maladie diagnostique relativement facile et est totalement évitable lorsque le traitement des femmes enceintes et du partenaire est effectué de manière adéquate (ARAUJO et al., 2006), ce qui fait que sa prévalence est généralement liée à un pouvoir économique déficient de fournir une santé de base de qualité, plus précisément, d'offrir des soins prénataux efficaces à la population (ARAUJO et al., 2006).

Cependant, le sous-développement n'expliquerait pas à lui seul la cause de l'Amazonas étant le principal état de croissance de la maladie dans la période observée, car par rapport à d'autres États de la région du Nord, c'est le meilleur PIB, de sorte que l'amélioration de la notification de la maladie dans l'État, pourrait expliquer le fait que la région du Nord a une forte prévalence de cette comorbidité. En 2016, le RC: 96425

Lien d'accès: https://www.nucleodoconhecimento.com.br/sante/dans-le-nord 
département de la santé d'Amazonas a lancé une campagne de décentralisation pour le dépistage rapide des maladies sexuellement transmissibles, auparavant superconcentrées en FMT (Heitor Vieira Dourado Tropical Medicine Foundation), les redistribuant aux quatre SAEs (Specialized Care Services) de la ville (BRASIL, 2016).

Toujours dans les perspectives qui cherchent à expliquer la forte incidence de la syphilis congénitale dans la région nord, le sous-développement est également lié à une population avec un niveau de sensibilisation plus faible, c'est-à-dire qu'il ne suffirait pas seulement de la disponibilité des soins prénataux, mais de la compréhension de la société sur l'importance de sa réalisation, ce qui aide à comprendre pourquoi il s'agit d'un problème de santé publique encore si présent dans la région nord du Brésil, la deuxième région brésilienne ayant le PIB par habitant le plus faible, juste depuis le Nord-Est (IBGE, 2016).

En ce qui concerne l'origine ethnique variable dans les cas enregistrés de syphilis congénitale (figure 3), plus de $90 \%$ des cas étaient d'origine ethnique mixte (race), qui est également le groupe ethnique le plus répandu dans la région du Nord, représentant $66,88 \%$ de la population de cette région du Brésil (IBGE, 2010).

Il est également important de faire un parallèle avec deux données recueillies dans la recherche, le pourcentage de mères infectées qui ont subi des soins prénataux est supérieur à 70\% au cours de la période observée (2014-2019) (figure 4), c'est-à-dire que la plupart des femmes de la région font ce suivi, ce qui devrait impliquer une incidence plus faible de la maladie. D'autre part, le nombre de partenaires non traités est élevé. La syphilis est une maladie de transmission sexuelle, où il est obligatoire le traitement du partenaire. Cela reflète une réalité difficile du Brésil dans la réalisation de soins prénataux efficaces, le manque de suivi satisfaisant du partenaire dans les consultations. L'un des principaux problèmes qui existe encore dans le contrôle des infections sexuellement transmissibles (IST) pendant la grossesse est l'approche du partenaire (DUARTE, 2007). En outre, les problèmes se posent avec la notification et continuent de tenter de les convaincre de la nécessité d'un diagnostic et d'un traitement adhérent (DUARTE, 2007). 
Un autre facteur est le niveau d'analphabétisme, seulement $0,9 \%$ étaient analphabètes et le pourcentage le plus élevé est celui des personnes qui ont terminé au moins l'école primaire (figure 5). Cependant, environ $8 \%$ de la population du nord du Brésil est analphabète, la deuxième pire région, juste depuis la région du Nord-Est (IBGE, 2019). Cette comparaison des données conduit à des questions sur la sousdéclaration, si le pourcentage le plus élevé de femmes atteintes de syphilis dans la région ont effectivement fait des études supérieures ou si le pourcentage qui demande des soins et a les cas signalés est la majorité instruite et celle qui a le moins d'éducation suit sans demander de soins et n'a pas la maladie signalée.

Des soins prénataux inadéquats empêchent la routine pour le diagnostic et un traitement efficace et précoce (ARAUJO et al, 2006). Les données sur le moment du diagnostic renforcent l'importance de soins prénataux efficaces pour lutter contre l'incidence de la syphilis congénitale, puisque la majorité d'entre eux ont reçu le diagnostic à l'accouchement ou au moment du curetage (figure 6).

\section{CONCLUSION}

En raison de la forte incidence des cas de syphilis congénitale, la région du Nord est importante au détriment des autres régions brésiliennes. Le sous-développement de la région est un facteur fondamental pour expliquer ce fait, lorsqu'il y a un déficit dans les secteurs publics, en particulier dans l'éducation et la santé, les preuves se reflètent dans la population. En ce qui concerne la syphilis congénitale, la plupart des femmes enceintes avaient un faible niveau d'éducation, compte tenu de ces données, compte tenu du manque d'information sur l'importance des soins prénataux, à la fois en raison du manque d'intérêt de la population et de la faible diffusion du système de santé publique. À titre d'exemple, l'État d'Amazonas, qui était l'État avec le taux de pourcentage le plus élevé de cas signalés au cours de la période analysée en raison d'une plus grande performance dans le dépistage des infections sexuellement transmissibles, cependant, la plus grande disponibilité des méthodes de dépistage, exclusivement, n'est pas synonyme d'une amélioration de l'incidence des cas de syphilis congénitale. 
En outre, le nombre de partenaires non traités est très pertinent, car la voie de transmission la plus courante de la syphilis est la voie sexuelle. II y a un manque d'information et de résistance de la part de la plupart d'entre eux. Ainsi, il est nécessaire que la femme enceinte et son partenaire soient dépistés pendant les soins prénataux afin d'éviter une éventuelle réinfection d'un patient traité ou un diagnostic précoce.

Par conséquent, il est observé que pour réduire l'incidence des cas de syphilis congénitale, une série de facteurs sont nécessaires, allant de la plus grande disponibilité d'informations sur l'importance des soins prénataux et leur continuité par les femmes enceintes et leurs partenaires. Par conséquent, couvrez le diagnostic et le traitement précoces, afin d'éviter des complications pour le fœtus ou le nouveau-né.

\section{LES RÉFÉRENCES}

ARAUJO, Eliete da Cunha; Costa, Kelly de Souza Gama; SOUZA E SILVA, Rafaela; AZEVEDO, Valéria Nascimento; Lima, Fábio André Souto. Importância do pré-natal na prevenção da Sífilis Congênita. Revista Paraense de Medicina, Belém, v. 20, n. 1 p. $47-51, \quad$ mar. 2006.2 Disponível em: http://scielo.iec.gov.br/scielo.php?script=sci arttext\&pid=S010159072006000100008\&lng=pt\&nrm=iso Acesso em: 24 maio 2019.

BARROS, Yara Lorrane Souza de. Et al. Numbers of confirmed syphilis cases in pregnant women in Brazil between 2009 and 2013. Revista Científica Multidisciplinar Núcleo do Conhecimento. Year 05, Ed. 11, Vol. 25, pp. 53-61. November 2020. ISSN:2448-0959, Access link in: https://www.nucleodoconhecimento.com.br/health/syphilis-cases, DOI: 10.32749/nucleodoconhecimento.com.br/health/syphilis-cases

BRASIL, Ministério da Saúde. Departamento de Doenças de Condições Crônicas e Infecções Sexualmente Transmissíveis: Manaus recebe apoio da ONG AHF para descentralizar o atendimento de HIV/aids. 2016. Disponível em: 
http://www.aids.gov.br/pt

br/noticias/manaus-recebe-apoio-da-ong-ahf-paradescentralizar-o-atendimento-de-hivaids _ Acesso em: 23 maio 2019._

BRASIL, Ministério da Saúde. Departamento de Vigilância, Prevenção e Controle das Infecções Sexualmente Transmissíveis, do HIV/Aids e das Hepatites Virais: Protocolo Clínico e Diretrizes Terapêuticas para Atenção Integral às Pessoas com Infecções Sexualmente Transmissíveis (IST). 2018. Disponível em: http://conitec.gov.br/images/Artigos Publicacoes/Diretrizes/PCDT Atencao Integral IST 22-10-18.pdf Acesso em: 23 maio 2019.

CARDOSO, Ana Rita Paulo; ARAÚJO, Maria Alix Leite; CAVALCANTE, Maria do Socorro; FROTA, Mirna Albuquerque; DE MELO, Simone Paes. Análise dos casos de sífilis gestacional e congênita nos anos de 2008 a 2010 em Fortaleza, Ceará, Brasil. Ciência \& Saúde Coletiva. v. 23, n. 2, p. 563-574, 2018. Disponível em: https://www.scielosp.org/article/csc/2018.v23n2/563-574/ Acesso em: 24 maio 2019.

CAVALCANTE, Ana Egliny S; SILVA, Maria Adelane M; RODRIGUES, Antonia Regynara M; NETTO, José Jeová Mourão; MOREIRA, Andréa CA; GOYANNA, Natália F. Diagnóstico e tratamento da sífilis: uma investigação com mulheres assistidas na atenção básica em Sobral, Ceará. DST Jornal Brasileiro de Doenças Sexuais. v. 24, n. 4, p. 245-245, 2012. Disponível em: http://www.dst.uff.br/revista244-2012/4-Diagnostico\%20e\%20Tratamento\%20da \%20Sifilis.pdf Acesso em: 23 maio 2019.

CAVAlCANTE, Patricia Alves de Mendonça; PEREIRA, Ruth Bernardes de Lima; CASTRO, Jose Gerley Diaz. Sífilis gestacional e congênita em Palmas, Tocantins, 2007-2014. Epidemiologia e Serviços de Saúde. v. 26, n. 2, p. 255-264, abr./jun, 2017. Disponível em: https://www.scielosp.org/article/ress/2017.v26n2/255264/pt/Acesso em: 23 maio 2019.

CRT. Centro de Referência e Treinamento DST/AIDS-SP. Coordenadoria de Controle de Doença, Secretaria de Estado da Saúde de São Paulo (SES-SP). Guia de bolso para o manejo de sífilis em gestante e sífilis congênita. 2 ed., 112 p. São Paulo: 
Secretaria de Estado da Saúde. 2016. Disponível em: http://www.saude.campinas.sp.gov.br/saude/doencas/sifilis/guiadebolsodasifilis_2edi cao2016.pdf Acesso em: 23 maio 2019.

DUARTE, Geraldo. Extensão da Assistência Pré-natal ao Parceiro como Estratégia de Aumento da adesão ao Pré-natal e Redução da Transmissão Vertical de Infecções. Revista Brasileira de Ginecologia e Obstetrícia. v. 29, n. 4, p. 171-174, 2007.

GUINSBURG, Ruth; SANTOS, Amélia Miyashiro Nunes dos Santos. Critérios diagnósticos e tratamento da sífilis congênita. Documento científico Departamento de Neonatologia. São Paulo: Sociedade Brasileira de Pediatria. 2010. Disponível

em:

https://www.sbp.com.br/fileadmin/user upload/2015/02/tratamento sifilis.pdf Acesso em: 24 maio 2019.

Instituto Brasileiro de Geografia e Estatística (IBGE). Contas regionais do Brasil: 2010-2014, Coordenação de Contas Nacionais. - Rio de Janeiro: IBGE, 2016. 97 p. (Contas nacionais, ISSN 1415-9813; n. 53). Disponível em: https://biblioteca.ibge.gov.br/visualizacao/livros/liv98881.pdf Acesso em: 24 maio 2019.

Instituto Brasileiro de Geografia e Estatística (IBGE), Diretoria de Pesquisas, Coordenação de Trabalho e Rendimento, Pesquisa Nacional por Amostra de Domicílios Contínua 2016-2018. 2019. Disponível em: https://biblioteca.ibge.gov.br/visualizacao/livros/liv101657_informativo.pdf Acesso em: 24 maio 2019.

IBGE. Instituto Brasileiro de Geografia e Estatística. Sistema IBGE de Recuperação Automática - $\quad$ SIDRA. 2010. Disponível em: https://sidra.ibge.gov.br/Tabela/3175\#resultado Acesso em: 24 maio 2019.

MOTTA, Isabella almeida; DELFINO, Isabella Rey de Souza; DOS SANTOS, Leticia Vettorazzi; MORITA, Maura Omori; GOMES, Rayanne Gonçalves Dantas; MARTINS, Talita Pouzas Soares; CARELLOS, Ericka Vianna Machado; ROMANELLI, Roberta RC: 96425

Lien d'accès: https://www.nucleodoconhecimento.com.br/sante/dans-le-nord 
Maia de Castro. Sífilis congênita: por que sua prevalência continua tão alta?. Revista Médica de Minas Gerais, Belo Horizonte, v. 28. Suppl. 6, 2018. Disponível em: http://rmmg.org/artigo/detalhes/2418 Acesso em: 23 maio 2019.

ROCHA, Karina Dias. Et al. Number of congenital syphilis cases in Brazil between 2009 and Revista Científica Multidisciplinar Núcleo do Conhecimento. Year 05, Ed. 05, Vol. 01, pp. 131-143. May 2020. ISSN:2448-0959 DOI: 10.32749/nucleodoconhecimento.com.br/health/congenital-syphilis-cases

Soumis : Décembre 2020.

Approuvé : Décembre 2020. 